\title{
LA MÉDIATION DU PATRIMOINE SOUS-MARIN : LES MOYENS D'UNE CYBER-EXPOSITION ET D'UNE ARCHÉOLOGIE PARTICIPATIVE SUR LA FOUILLE SUBAQUATIQUE DE QAITBAY, ALEXANDRIE, ÉGYPTE
}

\author{
ISABELLE HAIRY \\ isabelle.hairy@,cnrs.fr \\ CNRS - UMR 8167 ORIENT \& MÉDITERRANÉE - CENTRE D’ÉTUDES \\ ALEXANDRINES (CEALEX, USR 3134 CNRS) \\ http://dx.doi.org/10.25267/Riparia_sup.2018.i1.12
}

\begin{abstract}
Depuis vingt ans, l'archéologie alexandrine s'est en partie tournée vers la Méditerranée ${ }^{1}$. Les zones côtières sont devenues le terrain de fouilles privilégié des archéologues, révélant des sites exceptionnels, voire uniques, comme celui de Qaitbay $^{2}$ qui avait été repéré depuis la surface depuis plusieurs siècles, comme l'indique dans sa relation de voyage, Benoît de Maillet (1656-1738), qui fût consul général de France en Égypte de 1692 à $1708^{3}$.

En 1994, la première fouille sous-marine de sauvetage, conjointe de l'IFAO et du CEAlex, fut organisée par l'archéologue helléniste Jean-Yves Empereur ${ }^{4}$, assisté
\end{abstract}

${ }^{1}$ E. KHAlil \& M. M. ABD EL-MAguid, «Underwater archealogy in Egypt», International Handbook of Underwater Archaelogy, 2002, p. 519-534; I. DARWISCH \& M. M. ABD ELMAgUID, «Underwater archealogy in Egypt », Tropis VII, vol. II, Athènes, 2002, p. 881-892; M. EL SAYED, «Unterwasserarchäologie in Ägypten », in Archäologie im Mittelmeer, Darmstadt, 2013, p. 127-138. L'histoire de l'archéologie sous-marine en Égypte a été évoquée dans de nombreuses communications lors du colloque qui s'est déroulé à Alexandrie, du 31 octobre au 2 novembre 2016, pour le 20e anniversaire du Département d'Archéologie sous-marine d'Égypte: "Alexandria International Conference on Maritime and Underwater Archaeology ».

2 Sur Kamel Abul Saadat, l'inventeur du site sous-marin au début des années 1960, voir H. HALIM, «Kamel Abul-Saadat: a pioneer in Alexandrian underwater archaeology», Underwater Archaeology and Coastal Management, Focus on Alexandria, Unesco, Paris, 2000, p 4653 ; S. A. ORCOS, "Early discoveries of submarine archaeological sites in Alexandria", Underwater archaeology and coastal management, Focus on Alexandria, UNESCO, Paris, 2000, pl. 4 ; M. M. ABD EL-MAGUID, « $\mathrm{Al}$ athar el ghareka be Misr fi meaat am », 2e colloque de l'association des archéologues arabes, Le Caire, 2000, p. 1373-1392, fig. 3 ; M. M. ABD EL-MAGUID, «Les fouilles récentes du Phare d'Alexandrie », Tropis VI, 2001, p. 21-32; H. RIAD, «Récentes découvertes à Alexandrie ", Conférence de la Société Archéologique d'Alexandrie, 1964. Sur les travaux d'Honor Frost qui lui a fait suite, voir H. FrOST 1975: "The Pharos Site Alexandria - Egypt», IJNA 4, p. 126-130 et H. Frost (Unesco Expert), « Report and Recommandations On the Submerged Architecture and Statues at the Site of the Ancient Pharos Fort Kaï Bey, Alexandrie », rapport dactylographié »; s.d., 1-11.

${ }^{3}$ B. DE MAILLET, Description de l'Égypte contenant plusieurs remarques curieuses sur la géographie ancienne et moderne de ce païs, sur les monuments anciens, sur leurs maurs, les coutumes et la religion des babitants, sur le gowvernement \& le commerce, sur les animaux, les arbres, les plantes, \& co. Composée sur les Mémoires de M. Maillet ancien consul de France au Caire, par M. l'abbé Le Mascrier, Paris, 1735, p. 131.

${ }^{4}$ J.-Y. EMPEREUR, rapports de fouille dans Bulletin de Correspondance Hellénique de 1995 à 2002; «Égypte : Le site du Phare d'Alexandrie», Archéologia 311, 1995, p. 30-33; «On a retrouvé le Phare d'Alexandrie !», L'Histoire 187, avril 1995; «Alexandria : The Underwater Site near Qaitbay Fort », Egyptian Archaelogy 8, 1996, p. 7-10; «The Discovery of the Pharos in Alexandria », Minerva 7,1, 1996, p. 5-6; "Raising Statues and Blocks from the Sea at 
de deux amis égyptologues, Jean-Pierre Corteggiani ${ }^{5}$ et Georges Soukiassian ${ }^{6}$, sur le site où l'on plaçait traditionnellement l'ancien Phare d'Alexandrie'. Cyriaque d'Ancône (1391-1455), marchand fasciné par l'Antiquité, fut le dernier voyageur à avoir vu les ruines de la tour en $1435^{\circ}$, soit un peu plus de quarante ans avant la construction du fort mamelouk à son emplacement, alors que la tour était en ruine depuis quelques dizaines d'années? .

Le site s'étend au pied du fort de Qaitbay, fort médiéval construit par le sultan Al Ashraf Seif el Dine Qaitbay, en 1477 à la pointe Est de l'éperon rocheux qui termine l'ancienne île de Pharos, devenue presqu'île par les atterrissements de sable qui se sont accumulés de part et d'autre de l'Heptastade ${ }^{10}$ et du tombolo qui s'étendait entre l'île et le continent. Sous la conduite du CEAlex, les fouilles se penchent dès 1994 sur les vestiges de plusieurs aménagements immergés à cet emplacement, plusieurs milliers de pièces de style grec et de tradition pharaonique, parmi lesquels les ruines du Phare d'Alexandrie qui a rayonné sur la Méditerranée durant plus de quinze siècles ${ }^{11}$.

Alexandria », Egyptian Archaelogy 9, 1996, p. 19-22; «Les fouilles sous-marines du Phare d'Alexandrie ", Egypte, Afrique et Orient 6, sept. 1997, p. 2-8; "Alexandrie engloutie et sauvée », Muséart 76, nov. 1997, p. 95-98; La Gloire d'Alexandrie, catalogue de l'exposition du Petit Palais, mai-juillet 1998, Paris, 1998, p. 98-104; Le Phare d'Alexandrie, la Merveille retrouvée, Gallimard, Paris, 1998 et 2004; "Diving on a sunken city», Archaeology, March/April 1999, p. 37-43; "Underwater Archaeological Investigations of the Ancient Pharos ", in M.H. HASSAN, N. GRIMAL et D. NAKASHIMA (éd.), Underwater archaeology and coastal management: Focus on Alexandria, Coastal Managements sourcebooks 2, 2000, Paris, Unesco, p. 54-59; «Le phare d'Alexandrie », Les fabuleuses découvertes du XXe siècle, Dossarch 259, 2000, p. 144-150; «Les fouilles sous-marines du CNRS à Alexandrie (Égypte) 1 - Le site monumental de Qaitbay", Tropis VII : 7th International Symposium on Ship Construction in Antiquity (Pylos 1999), Athènes, 2002, p. 325-334 ; Alexandrie redécouverte, Fayard, Paris, 2004, p. 63-87; J.-Y. EMPEREUR et N. GRIMAL, «Les fouilles sous-marines du phare d'Alexandrie », CRAI 1997, p. 693-713. A.-M. GUIMIER-SORBETS et J.-Y. EMPEREUR, « Le phare d'Alexandrie », Historia 606, juin 1997, p. 62-65.

${ }^{5}$ J.-P. CORTEGgianI, «Les Ægyptiaca de la fouille sous-marine de Qaïtbay », Bulletin de la Société française d'Égyptologie 142, juin 1998, p. 25-40 ; J.-P. CORTEGGIANI, " La mèche de Ptolémée », Historia thématique, 69, janvier-février 2001, p. 18-19.

${ }^{6}$ L'égyptologue Georges Soukiassian prépare la publication d'un volume sur les Ægyptiaca du site immergé de Qaitbay qui paraîtra dans la collection des Études Alexandrines.

${ }^{7}$ La construction du Phare d'Alexandrie a été lancée au début du III siècle av. n. è. par Ptolémée Ier Sôter (305-283 av. n. è.) et achevée par son fils, Ptolémée II Philadelphe (283246 av. n. è.) une quinzaine d'années plus tard.

${ }^{8}$ Il rapporte sa visite aux ruines du Phare dans un ouvrage publié à Florence en 1742, Kyriaci Anconitani Itinerarium.

${ }^{9}$ Ibn Battûta qui visita Alexandrie par deux fois, note au cours de son second voyage en 1349 que la porte du Phare n'est plus accessible.

10 Chaussée construite durant l'Antiquité mesurant 7 stades de long et qui joignait le continent à l'île de Pharos. Voir les travaux de A. Hesse sur la recherche de son tracé sur la presqu'île: "L'Heptastade », La gloire d'Alexandrie, Paris, 1998, p. 88-89, et "L'Heptastade d'Alexandrie », Alexandrina, Études alexandrines 6, Le Caire, 2002, Vol. 6, p. 191 - 273.

${ }^{11}$ Le 8 août 1303 (702 de l'Hégire), un formidable tremblement de terre touche l'Est de la Méditerranée, et le Phare subit de gros dommages qui ne seront jamais réparés. Selon le Petit Thalamus de Montpellier, un cartulaire occitan du XIII e siècle (in Thalamus Parvus, chronique romane des XIII/XVII siècles, publiée en 1836), le séisme date la fin de l'utilisation de la tour signal qui est laissée à l'abandon. Durant plus d'un siècle, la tour finira de se disloquer 
Le site est vaste et c'est là une de ses plus grandes difficultés. Géographiquement, il s'étend sur plus de 3 hectares de grès dunaire et de sable. La partie visible des 3032 blocs $^{12}$ répertoriés dans les bases de données début 2017, gisant par 2,60 m à $9 \mathrm{~m}$ de fond se développe sur plus de $13000 \mathrm{~m}^{2}$ (fig. 1).

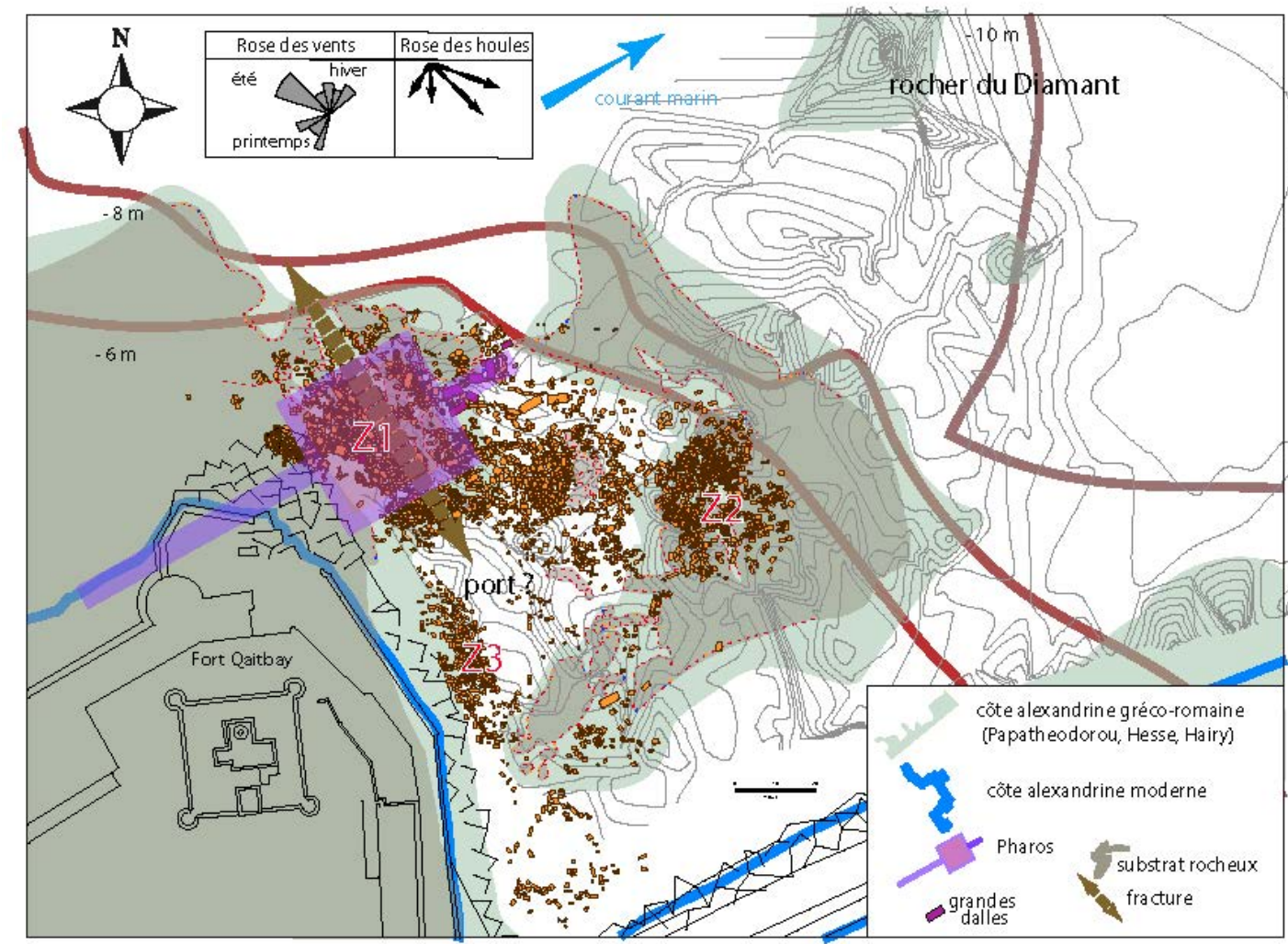

Fig.1. Carte du site immergé de Qaitbay avec emplacement supposé de l'ancien Phare d'Alexandrie, et indication des trois zones correspondant à des aménagements différents: $Z 1=$ blocs du Phare, $Z 2=$ aménagement époque romaine \& $Z 3=$ ruines d'une jetée médiévale faites de blocs antiques (carte I. HAIRY).

On évalue le nombre total de blocs autour de 3500 en raison des zones ensablées et des amoncellements de blocs en contre-bas des platiers. Les pièces enregistrées sont classées en quatre grandes catégories: blocs architecturaux, statuaire, blocs appartenant à un autre type de monument (naos, obélisque, vasque, etc.) et objets métalliques (plomb, fer et bronze), appartenant principalement à des scellements architecturaux. Les contraintes de l'étude des pièces immergées sont diverses : difficulté d'accès aux pièces sous l'eau, problème de visibilité, de pollution qui va grandissant avec l'accroissement de la population d'Alexandrie, - même si depuis quelques années, des mesures ont été prises dans le sens d'une amélioration -, obstacles liés au poids des pièces, - les plus importantes faisant plus de 40 tonnes. Les renflouages réalisés entre 1995 et $1999^{13}$ ont vite montré les limites d'une telle

sous le choc des vagues, et sous les outils des tailleurs de pierre venus récupérer de la matière première, jusqu’à la dernière mention de la présence des ruines en 1435.

${ }^{12}$ Cela correspond à une masse d'environ 5256 tonnes de roches importées, parmi lesquelles on trouve $76,09 \%$ de granite.

${ }^{13}$ Entre 1995 et 1996, une trentaine de pièces majeures du site archéologique sous-marin furent renflouées. Hormis les deux fragments du piedroit d'une porte monumentale restés 
action : l'investissement financier que représente le coût du renflouage et le travail engendré par le traitement des blocs avant de pouvoir les exposer à l'air libre après des siècles d'immersion, générant de nouvelles dépenses de moyens et une durée conséquente des traitements de dessalage. Il faut trouver ensuite de nouveaux moyens techniques et financiers pour la restauration, la conservation et l'entretien des espaces où ces pièces sont exposées, pour des résultats somme toute assez limités, puisque dans le domaine scientifique, seules trois anastyloses partielles ont pu être menées à partir des fragments renfloués. 36 pièces ont été renflouées ${ }^{14}$ sur les 3500 repérés sous l'eau, soit tout juste $1 \%$ des ruines immergées. L'accès à ce patrimoine parmi les plus riches de l'Alexandrie antique n'est pas satisfaisant, même si certains monuments, comme la statue colossale d'un roi ptolémaïque, statue emblématique puisque certains l'identifie au fondateur de l'ancien museion dont faisait partie la première Bibliothèque, ont pu grâce à ce travail pharaonique rejoindre la postérité de la ville moderne ${ }^{15}$ (fig. 2).

après leur renflouage sur le quai ouest du port est et les pièces du colosse exposé devant la Bibliotheca Alexandrina, toutes les pièces ont été traitées et sont aujourd'hui exposées sur le site archéologique de Kom el-Dikka dans un musée de plein-air. En 2000, le Service des Antiquités Egyptiennes a décidé de ne plus autoriser le renflouement des pièces de plus de $100 \mathrm{~kg}$. Le Conseil Suprême des Antiquités fera tout de même une exception pour une main droite de statue, remontée sur dérogation en 2001 afin d'être restituée à son propriétaire dans le cadre du programme de recherche européen Médistone. Elle a pu être replacée sur le colosse représentant un des deux premiers Ptolémée exposé à présent devant la Bibliotheca Alexandrina.

1434 pièces ont été renflouées en 1995 et 2 autres pièces les ont rejoint en 1996, - la tête du colosse d'un roi grec en pharaon et un sphinx en granite -, suite à la visite à Alexandrie de l'ancien président français, Jacques Chirac.

15 Les quatre fragments de la statue colossale, une fois renfloués, traités et conditionnés, avaient été transportés à Paris pour restauration dans le cadre d'un contrat passé avec la France pour l'assemblage des fragments du colosse. C'est EDF, au travers d'un mécénat technologique et scientifique, qui était en charge de l'accostage des fragments. La statue remontée a été exposée en 1998 devant le Musée du Petit Palais à Paris, durant l'exposition «La gloire d'Alexandrie », puis à Agde la même année, avant de revenir en Égypte. À son retour, elle a été érigée devant l'entrée de la Bibliotheca Alexandrina, rendant ainsi hommage au fondateur de la cité telle qu'elle rayonna sur la Méditerranée jusqu'à la conquête romaine. 


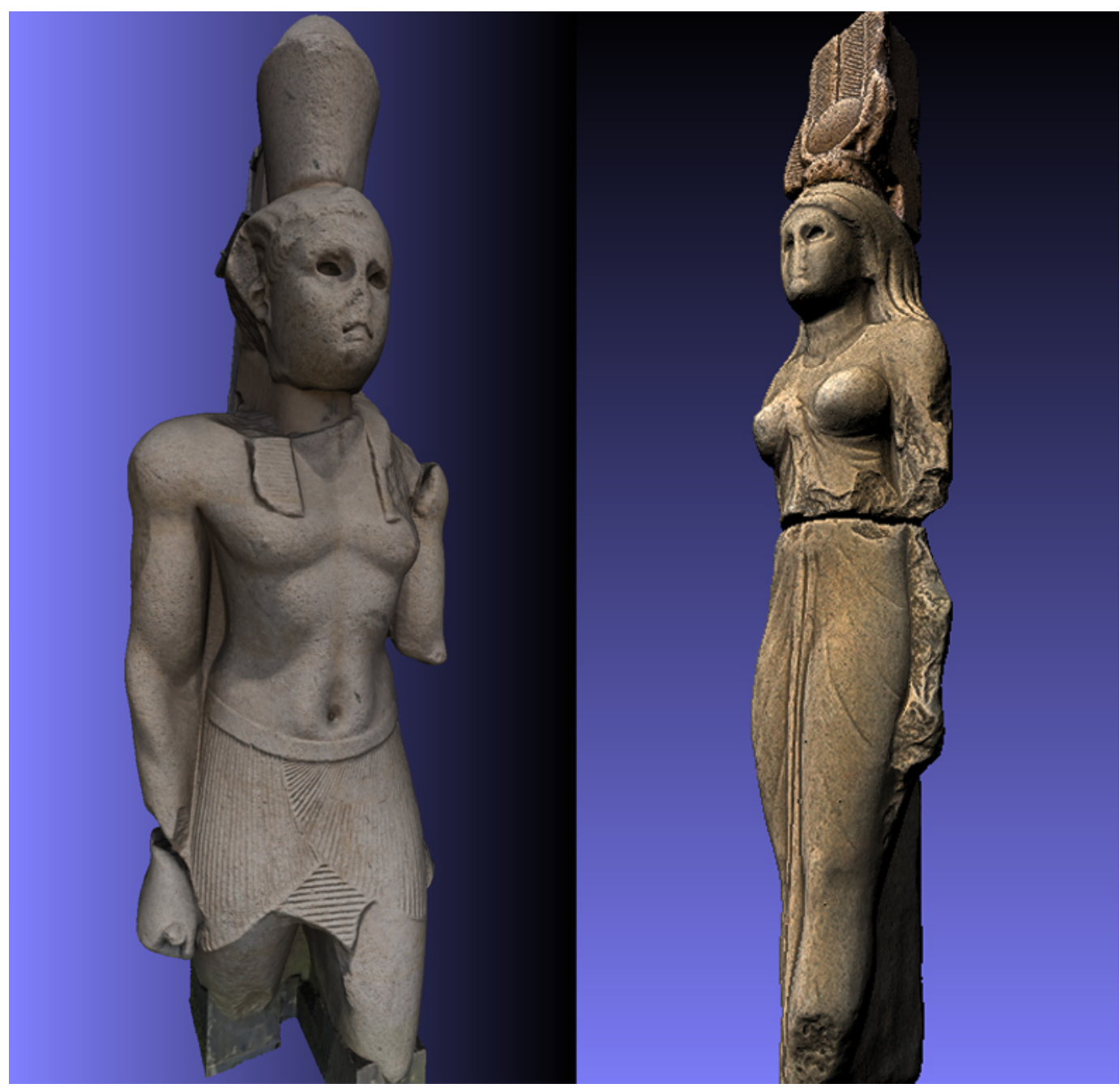

Fig. 2. Doubles numériques des statues colossales d'un roi grec en pharaon et d'une reine grecque en Isis (photographies A. HuSSEIN GOMA et Ph. SOUBIAS, photogrammétrie M. ABD EL AZIZ, restitution virtuelle (Isis) et DAO I. HAIRY).

À travers cette rapide présentation, on aura saisi l'importance d'un tel site pour le patrimoine alexandrin et égyptien, puisque le Phare d'Alexandrie est au moins aussi réputé que les pyramides de Chéops et Kephren! Mais il faut bien reconnaitre que la difficulté d'accès au site sous-marin rend sa visibilité quasi nulle pour le grand public, si ce n'est au travers d'images, de photos publiées dans des revues grand public, et de documentaires qui deviennent vite obsolètes à mesure que le travail des archéologues avance.

\section{Les moyens d'une cyber-exposition}

Aujourd'hui, on s'intéresse toujours autant au Phare et plus encore à son image. En faisant une exploration sur le web, j’ai trouvé des centaines de dessins représentant le Phare. J'ai noté que les restitutions de Thiersch restent la référence par laquelle les auteurs néophytes brodent au gré de leur imagination. Malgré tout, certaines illustrations s'inspirent déjà de nos travaux; elles se sont nourries aux idées nouvelles qui émergent au fur et à mesure que nous avançons dans la compréhension des ruines immergées, images arrêtées témoignant d'un état des travaux de la recherche. Ceci dit, on voit bien l'importance de communiquer au grand public les résultats de la fouille, de rendre le site accessible au plus grand nombre, en bref de démocratiser l'accès aux données. Mais sous quelle forme faut-il le faire ? 
Selon une étude réalisée par l'institut de sondage Ipsos et commandée par l'INRAP, 43\% des français sont intéressés par l'histoire et l'archéologie et 70\% d'entre eux ont visité au moins un site archéologique en France au cours de leur vie ${ }^{16}$. Cette étude montre par ailleurs que $77 \%$ des intéressés s'estiment insuffisamment informés sur les fouilles conduites près de chez eux. Il y a donc une véritable attente de la part du public séduit par l'archéologie; la demande d'information est forte. L'INRAP confirme ces données dans la pratique des journées «portes ouvertes» sur les chantiers de fouille qui sont marquées par une fréquentation importante et un public diversifié, parfois très éloigné de la culture savante. Les visiteurs s'intéressent à ce qu'ils perçoivent comme leur passé, leur histoire. Il serait bien entendu enrichissant de faire un sondage similaire en Égypte où les gens sont particulièrement fiers de leur patrimoine.

L'intérêt pour la présentation des vestiges in situ est donc réelle, d'autant plus forte si la fouille se déroule dans un caractère d'urgence ou de sauvetage, et que les vestiges sont appelés à disparaître. Il apparaît également que le profane est en quête d'explications sur les sites qu'il visite, à même de l'aider à comprendre le contexte de la fouille et son interprétation qui lui échappent bien souvent, à l'instar de ce qui est déjà pratiqué pour d'autres sciences. On ne peut cependant que constater qu'une présentation in situ d'une fouille d'urgence reste très éphémère, et si par chance, la mise en valeur est pérennisée par la conservation du site, elle ne touche bien souvent qu'un public de proximité.

Par ailleurs, on voit bien que le web et les supports nomades ont aujourd'hui un impact très important sur la vie sociale. Alors comment les utiliser pour transformer et pour renouveler les interactions entre la recherche archéologique et la société ? Et si l'on resserre la question autour du site qui nous intéresse ici : quelle représentation, quelle image l'archéologie sous-marine à Qaitbay peut-elle donner d'elle-même via les technologies numériques et le web ?

Les travaux menés sur le site sous-marin de Qaitbay présentent une large diversité, jusqu'à la photogrammétrie $3 \mathrm{D}$ qui est en train de révolutionner l'archéologie sous-marine. Les contenus numériques sont de plus en plus présents, jusqu'aux restitutions en réalité virtuelle qui pourront devenir le support à une adaptation nécessaire de la valorisation scientifique par lesquelles son périmètre d'action sera élargi. Alors concrètement, comment cette valorisation via le web et les supports nomades peut-elle être mise en œuvre et dans quel but?

La recherche s'appuie déjà depuis de nombreuses années sur les applications développées autour des contenus numériques ${ }^{17}$. Des supports de valorisation ont par la suite été développés, destinés à porter le programme de cette recherche, tout d'abord dans une relation unilatérale : émetteur-récepteur. Les cyber-musées ont sans doute été une des premières applications du partage d'éléments du patrimoine

16 Sondage effectué en 2010 auprès de 1000 personnes de plus de 14 ans. Parmi les 43\%, $19 \%$ se passionnent plus particulièrement pour l'archéologie, un pourcentage équivalent à celui de l'astronomie ou de la philosophie; Voir l'article sur le lien suivant (consultation mai 2017) : http://www.inrap.fr/les-francais-et-l-archeologie-un-sondage-d-ipsos-sur-l-image-del-archeologie-5093. Le rapport complet de l'IPSOS est en ligne à cette adresse : http://www.ipsos.fr/sites/default/files/attachments/image de larcheologie aupres du gra nd public.pdf.

17 Sur la fouille sous-marine de Qaitbay, les premiers essais en photogrammétrie ont été menés en 1999 par Sébastien Erome, sur des blocs de grandes dimensions et à partir de photos argentiques numérisées. I. HAIRY, Pharos, Chantier sous-marin du site de Qaitbay, Campagne de printemps et d'automne, rapport de travail, mai-juin \& octobre-novembre, 1999, p. 10. 
destinées à un large public, sous la forme de contenus numériques. Les exemples sont multiples dans ce domaine ; un projet de ce type avait été envisagé, il y a quelques années, pour communiquer sur la recherche autour du site de Qaitbay ${ }^{18}$, projet motivé par la multitude des données et leur dispersion de par le monde. Avec l'essor de la 3D, ce projet mériterait d'être relancé.

Cette valorisation à relation unilatérale trouve d'autres applications au travers de divers supports numériques dont nous pourrions nous inspirer pour communiquer sur l'archéologie subaquatique et la recherche autour du Phare.

En navigant sur le web, on découvre qu'un nombre important de projets de ce type voient le jour, des projets en libre accès, comme le très bel exemple du programme de valorisation Truelle et Pixels ${ }^{19}$ issu d'une collaboration entre la Maison de l'Orient et de la Méditerranée (MOM) de Lyon II et le CNRS qui tend à partager l'expérience de professionnels des métiers et des sciences de l'archéologie au travers d'un site web documentaire qui comprend de la photo et de la video, et s'est enrichi, dans un second temps, d'un simulateur 3D de chantier de fouilles apportant une expertise sur les compétences dans les métiers de l'archéologie et des activités pédagogiques pour les plus petits. Il faut également mentionner le projet européen de simulateur archéologique grand public, Simulex'Achéo ${ }^{20}$, qui s'appuie sur des simulations réalisées à partir de terrains réels numérisés, comme celui de Lattes, et qui devait être mis à disposition gratuitement sur divers supports. Son objectif était de fédérer une communauté de jeunes passionnés de l'archéologie et d'offrir aux plus performants d'entre eux une expérience en live sur un des sites partenaires du projet $^{21}$. Je n'ai malheureusement pas trouvé trace de la mise en ligne de cette application qui était prévue pour 2014. D'autres applications plus modestes sont gratuites et disponibles sur tablette, comme Archeo-palmus qui est issue d'un projet conçu par le Musée archéologique de la ville de Saint Raphaël et qui s'adresse aux enfants. L'application mobile se télécharge sur tablette avant la visite du musée afin que les enfants puissent profiter d'une visite personnalisée avec jeux immersifs et éducatifs leur apportant des connaissances techniques et historiques dans le domaine de l'archéologie sous-marine.

Terrains virtuels inspirés de sites réels, apprentissage de compétences par le biais de simulateurs sont les outils communs à ces applications. Leur objectif, le partage d'éléments du patrimoine situés sur le domaine public, en accès libre, exploités dans un environnement collaboratif.

La tendance actuelle est à l'ouverture vers une approche participative amenant les amateurs passifs de l'archéologie à devenir actifs à l'aide de moyens numériques, en prenant des photos par exemple ou en faisant des observations qui viendront compléter des bases de données interfacées avec différents outils, comme Géoportail. Il s'agit de modifier la relation unilatérale, émetteur-récepteur, recherche-

18 Le projet nommé Virtual Pharos Project, avait été imaginé par I. Hairy et B. Madran (Tetrazon).

19 Projet porté par la MSH MOM (USR 3439) et le laboratoire Archéologie des sociétés méditerranéennes (UMR 5140). Le site se visite à cette adresse (consultation mai 2017) : http://www.truelles-pixels.mom.fr/

${ }^{20}$ Projet soutenu par le Ministère de la Culture et par le Labex Archimede. Voir le descriptif $\mathrm{du}$ projet à l'adresse suivante (consultation mai 2017) : http://archimede.cnrs.fr/index.php/liens/118-projets-en-cours/projets-en-cours-aap1/169programme-simulex.

${ }^{21}$ (consultation mai 2017): http://www.20minutes.fr/montpellier/1139207-20130417premier-simulateur-fouilles-archeologiques. 
public, comme on la trouve encore dans des projets comme celui de Digital Karnak $^{22}$, pour prendre un exemple égyptien. Développé par l'UCLA (University of California à Los Angeles), sous la direction de Diane Favro et de Willeke Wendrich, l'application offre une visite très documentée, - photos, plans, restitutions -, du complexe des temples de Karnak, reconstruit et développé pendant plus de deux millénaires, ainsi qu'une découverte de l'histoire de ce site qui fut le centre religieux le plus étendu de toute l'Antiquité.

Ces systèmes ont évolué en mettant en scène des corpus de données archéologiques dans le cadre de systèmes de médiation, de valorisation numérique et collaboratifs, d'une part à vocation pédagogique et d'autre part à vocation de recherche participative.

Le jeu sert bien souvent de support à la vocation pédagogique de ces systèmes. Le processus de simulation sert à l'auto-apprentissage de la démarche scientifique; l'utilisateur exploite les données archéologiques réelles mises à sa disposition pour expérimenter les techniques qu'il doit approcher.

Sur le site sous-marin de Qaitbay, une série d'applications ludiques s'appuyant sur le travail des plongeurs-archéologues pourrait être proposée afin d'appréhender les techniques et les méthodes utilisées dans le cadre de la fouille: comme le calcul du volume d'un ballon pour déplacer un bloc de granite sous l'eau (fig. 3).

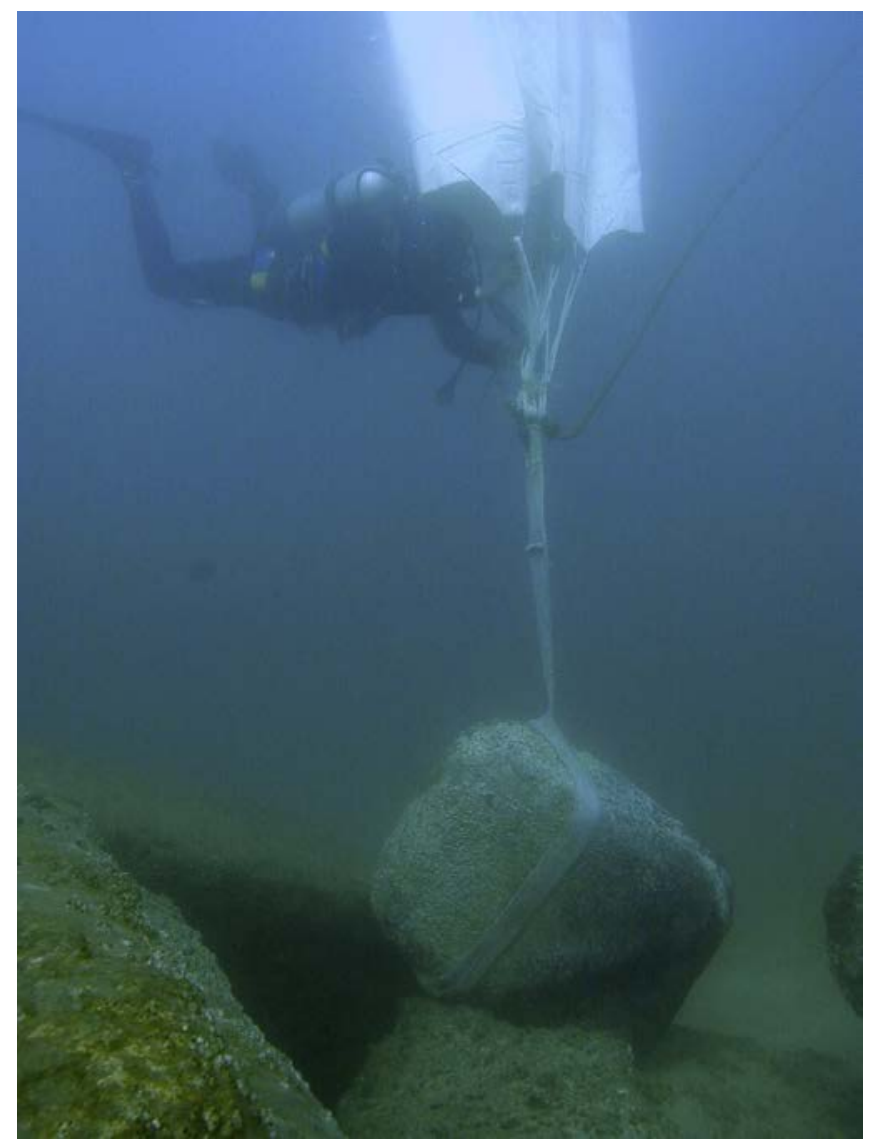

Fig. 3. Déplacement d'un bloc à l'aide d'un ballon (CEAlex, photo A. HusSEIN GOMA).

22 Voir le site à l'adresse suivante (consultation mai 2017) :
http://dlib.etc.ucla.edu/projects/Karnak/.


L'utilisateur aurait accès aux données réelles afin de réaliser l'exercice virtuel, dans une application du principe d'Archimède qui ouvre au passage, une fenêtre sur les sciences anciennes et l'histoire de l'école des ingénieurs alexandrins. Une autre application du travail sous-marin pourrait consister à nettoyer de façon virtuelle un bloc choisi par l'utilisateur lors de son exploration du site virtuel (fig. 4) pour en faire la description détaillée. L'accent serait mis sur les capacités d'observation de l'utilisateur; à l'issue de l'exercice, il aurait la possibilité de comparer son travail avec les observations réelles et de s'informer sur certains aspects de la description, comme les différents types de roche importée rencontrés sur le site, leurs origines, etc. en accédant à la banque numérique d'échantillons de roche prélevés sur le site.

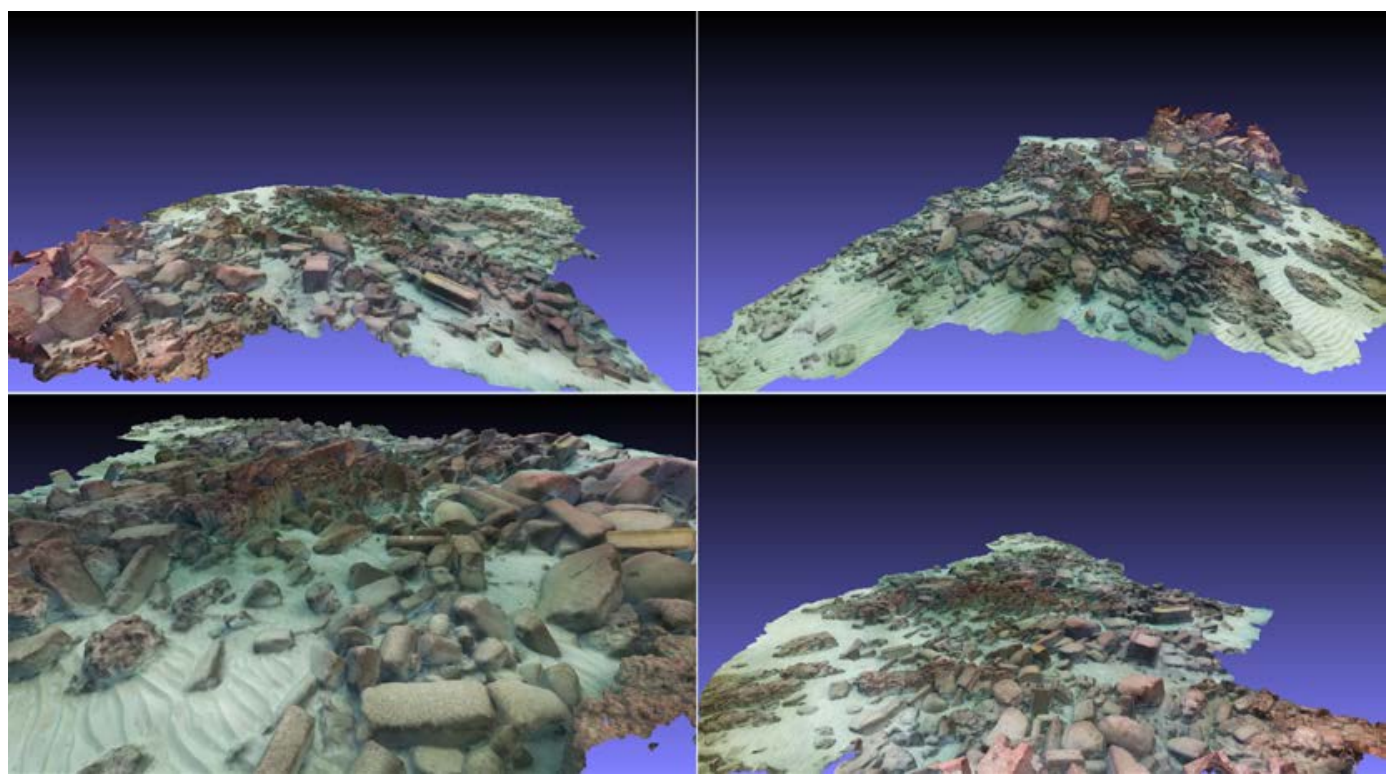

Fig. 4. Modèle numérique de surface du site sous-marin de Qaitbay avec vue sur la zone des blocs du Phare (CEAlex, photographies M. EL SAYED, photogrammétrie M. ABD EL AZIZ, DAO I. HAIRY).

Les plombs de scellement pourraient également faire l'objet d'un exercice : les retrouver sur le site, les identifier, les décrire, les renflouer, les restaurer, les étudier.

On voit bien la multitude de processus de simulation qui peuvent être imaginés autour des activités réelles pour initier l'utilisateur aux métiers, aux techniques et à l'approche scientifique de l'archéologie subaquatique. Il s'agit d'appréhender le site sous un angle pratique, sur le mode de la simulation, donc sans danger, sans compétences de la plongée, et surtout avec le droit à l'erreur.

En matière de libre accès et de partage des données, il faut encore citer le très bel exemple du projet Karnak, lancé en janvier 2013 et qui regroupe plusieurs acteurs $^{23}$. Il a pour objectif de rendre accessible une documentation textuelle exhaustive issue des temples de Karnak, liée à un moteur de recherche permettant des requêtes sur le contenu des notices et dans les inscriptions hiéroglyphiques par le biais de la translittération. Il est étroitement lié au programme VÉgA (Vocabulaire de

23 CNRS, USR 3172 - CFEETK / UMR 5140, Équipe ENiM - Programme « Investissement d'Avenir » ANR-11-LABX-0032-01 Labex Archimede. 
l'Égyptien Ancien) qui a été mis en ligne récemment ${ }^{24}$, anciennement DPEA (Dictionnaire Permanent de l'Égyptien Ancien), placé sous la direction scientifique de Frédéric Servajean, et qui a pour objectif la réalisation d'un outil en ligne, avec une mise à jour quotidienne, s'appuyant sur le premier dictionnaire général numérique de l'égyptien ancien en langue française depuis le déchiffrement des hiéroglyphes par Champollion en 1822.

Voyons à présent la vocation de recherche participative de ces systèmes de médiation et de valorisation numériques et collaboratifs ${ }^{25}$. Les programmes d'archéologie participative fleurissent depuis quelques années. Ils ont généralement pour objectif d'utiliser un public averti pour l'inventaire d'un patrimoine en danger. L'amateur passif devient acteur de certains aspects de la recherche. Je citerais un très bel exemple d'archéologie participative: le projet Alert, sentinelle du patrimoine côtier regroupant des archéologues, des géographes et des géomorphologues. Les amateurs bénévoles adhérents à ce programme sont des acteurs extérieurs qui produisent de la donnée sans l'interpréter. Ils alimentent une plate-forme de renseignements web et une application mobile qui permettent d'alerter les scientifiques de la découverte de sites archéologiques mis au jour par l'érosion du littoral. Ils enregistrent des observations, - avec notes et photos -, qui permettront de mettre éventuellement en place une fouille d'urgence. On rêverait d'un tel système en Égypte, et plus particulièrement sur les côtes alexandrines !

Le dernier aspect des sciences participatives transforme plus profondément les interactions entre la recherche et la société. Dans cette dernière phase, on appréhende plus complètement de quelle façon le contenu éditorial peut être impacté par les innovations numériques et par les effets de réseau dans un monde où de plus en plus d'internautes investissent les sciences participatives. En matière d'enrichissement de biens communs, l'encyclopédie non commerciale Wikipédia, créée en 2001, comptait en 2011 pas moins de 30 millions de contributeurs de par le monde; en 2016, le nombre de contributeurs actifs français était de 16959 . Le CNRS et le CNAM en ont fait le sujet d'un colloque en 2013 intitulé «Wikipédia, objet scientifique non identifié $»^{26}$. Cependant, la communauté scientifique s'interroge sur la crédibilité et l'exactitude de la connaissance de l'encyclopédie. Et la question est loin d'avoir trouvé des réponses satisfaisantes en raison de la difficulté à mener les comparaisons. Si la réponse ne peut être globale, il semble en revanche qu'elle puisse être posée au cas par cas : on regarde si un article est biaisé ou fiable.

Parlons maintenant d'un projet plus scientifique qui rassemble 28000 membres ${ }^{27}$ participant à des programmes du CNRS ou du Musée national d'histoire naturelle (MNHN) sur l'observation de la nature: il s'agit de l'association TelaBotanica $^{28}$, réseau des botanistes francophones. L'objectif du réseau est de favoriser l'échange d'informations et de produire des données libres de droit au service de l'ensemble des botanistes, en s'appuyant sur les nouvelles technologies de la

${ }^{24}$ L'ouverture au public a été faite le 16 mai 2017, dans le cadre du salon Innovatives SHS 2017. http://vega-vocabulaire-egyptien-ancien.fr/ (consultation mai 2017).

25 À ce propos, voir le rapport daté du 4 février 2016, les Sciences participatives en France, état des lieux, bonnes pratiques \& recommandations, fev 2016, commandé par le Ministère de l'éducation nationale de l'enseignement supérieur et de la Recherche.

26 Voir: http://www.lemonde.fr/sciences/article/2016/01/11/wikipedia-un-objetscientifique-non-identifie 4845331 1650684.html. (consultation mai 2017)

${ }^{27}$ Données 2017.

28 Adresse du portail d'entrée du site web (consultation mai 2017) : http://www.telabotanica.org/site:accueil. 
communication. La communauté des telabotanistes vient en fait combler un vide laissé dans l'enseignement et la recherche : celui de la botanique fondamentale ou de terrain.

On en vient donc à s'interroger sur le danger du développement de tels projets qui pourraient à plus ou moins court terme aider à faire disparaitre des pans entiers de la recherche. Mais dans quel sens le processus fonctionne-t-il réellement ?

Ces collaborations étroites entre professionnels de la recherche et nonprofessionnels tendent à accélérer les travaux de collecte des données; elles aident également à créer de nouveaux savoirs qui aboutissent parfois à de réels progrès scientifiques, comme pour le projet Galaxy Zoo ${ }^{29}$ créé en 2006 et lancé en 2007, et pour lequel des milliers de volontaires ont observé des images du ciel générés par divers télescopes avec des résultats étonnants. Les champs disciplinaires concernés sont divers : conservation des archives, biodiversité, biologie, patrimoine, etc.

Le contexte propice à ces programmes est le suivant: des données massives qui se heurtent au manque de moyens humains et financiers, et à l'absence de reconnaissance pour les activités d'enrichissement des bases de données.

Mettre en place une coopération entre scientifiques et contributeurs citoyens exige du temps et une forte organisation, malgré tout, les expériences collaboratives en sciences participatives se développent. Je citerais un exemple qui concerne directement l'Égypte et qui se rapproche de la problématique du site sous-marin de Qaitbay: il s'agit du projet de l'université d'Oxford pour la traduction des papyri d'Oxyrhynque ${ }^{30}$ où il fallait rendre accessibles à une communauté d'amateurs éclairés au travers d'une plate-forme web des jeux de données, en l'occurrence 500000 fragments de papyri retrouvés en Égypte en 1897. En un siècle, les scientifiques avaient traduit 1\% des papyri. En trois ans de web opération, plus de 100000 papyri ont été décryptés et pour certains traduits. Ce projet monté par le papyrologue anglais Dirk Obbink, de l'université d'Oxford, montre à quel point un tel projet peut être moteur de la recherche.

Avec près de 3500 blocs repérés sous l'eau parmi lesquels 3036 sont enregistrés dans les bases de données, nulle doute que le site de Qaitbay puisse être l'objet du développement d'un programme d'archéologie participative sous la forme d'une plate-forme web présentant plusieurs stratégies de valorisation et de recherche :

- un cyber-musée présentant les sources documentaires sur le site et sur le Phare d'Alexandrie, une banque virtuelle de copies numériques 3D des blocs du site, ainsi qu'une visite guidée du modèle numérique de surface (MNS) du site sous-marin, autant de données numériques évoluant au rythme des découvertes,

- des applications pédagogiques avec différents contextes d'apprentissage donnant accès à certaines données réelles: initiation aux métiers, aux techniques et à l'approche scientifique de l'archéologie subaquatique,

- enfin, un dispositif de sciences participatives à partir d'un réseau d'internautes avertis, sélectionnés, ayant un accès libre à certaines données afin d'accélérer leur traitement: observation du site au travers des jeux d'orthophotoplans en

29 Adresse du portail d'entrée du site web (consultation mai 2017) : https://www.galaxyzoo.org/?lang=fr.

30 Voir à cette adresse, le portail d'entrée du site (consultation mai 2017) : http://www.papyrology.ox.ac.uk/POxy/. 
HD ou UHD, création de doubles numériques 3D en photogrammétrie des nombreux blocs immergés à partir de jeux de photos mis à disposition, etc.

Avec l'utilisation de la photogrammétrie par corrélation d'images denses et de la 3D dans la recherche archéologique sur le site sous-marin de Qaitbay, de nouvelles pistes méthodologiques sont apparues, aussi bien dans les pratiques de terrain où les méthodes modernes se sont substituées aux méthodes traditionnelles, que dans les études consacrées au mobilier immergé. Ces nouveaux usages ont entraîné de profondes transformations dans les pratiques d'investigations: la manipulation de données dans un environnement tridimensionnel, la capacité de corriger la visualisation des surfaces des objets, la capacité de produire des restitutions scientifiques en 3D (fig. 5) peu figées et de représenter virtuellement les environnements disparus, etc. Ces nouveaux usages du numérique nous mèneront inexorablement à ouvrir de nouvelles portes vers les domaines de la valorisation et vers une approche participative de la recherche.

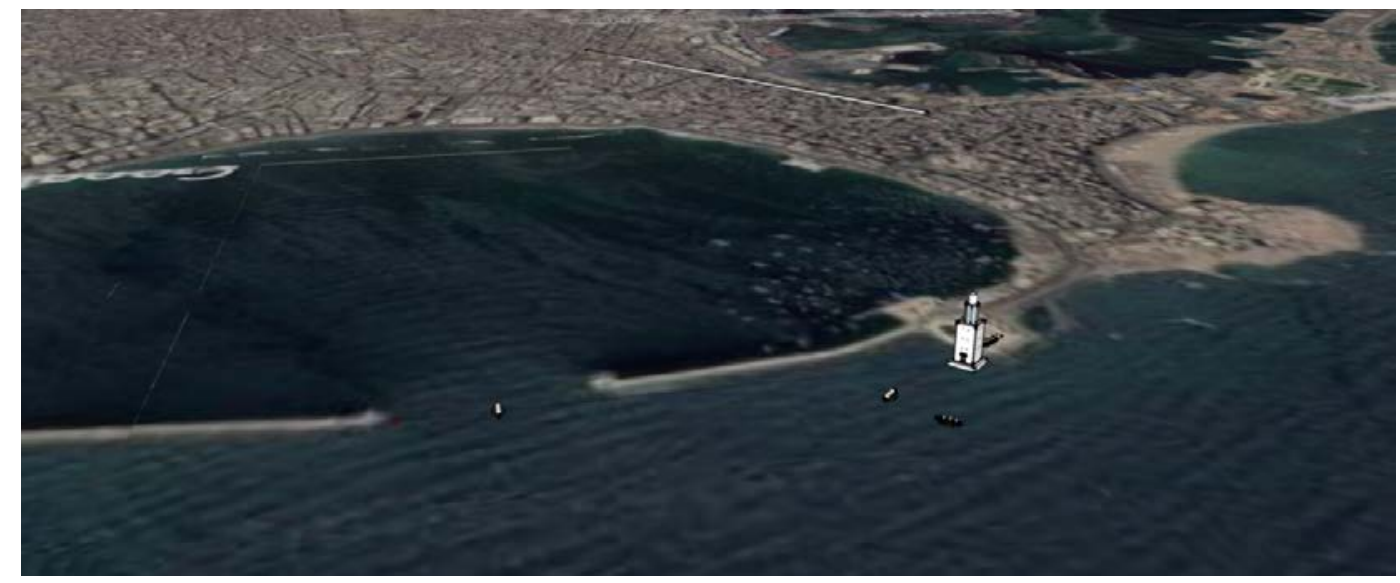

Fig. 5. Restitution du Phare d'Alexandrie avec insertion dans le site (3D I. HAIRY). 